\title{
LINEAR GROUPS OF FINITE COHOMOLOGICAL DIMENSION
}

\author{
BY ROGER C. ALPERIN ${ }^{1}$ AND PETER B. SHALEN ${ }^{1}$
}

Our main result provides necessary and sufficient conditions for a finitelygenerated subgroup of $G L_{n}(\mathrm{C}), n>0$, to have finite virtual cohomological dimension. A group has finite virtual cohomological dimension (VCD) if it has a subgroup of finite index which has finite cohomological dimension; this dimension is, in fact, the same for all torsion-free subgroups of finite index. It is, of course, necessary for a group $\Gamma$ with $\operatorname{VCD}(\Gamma)<\infty$ to have torsion-free subgroups of finite index; this is guaranteed in the case of finitely-generated linear groups by a well-known result of Selberg which extends ideas of Minkowski.

A subgroup of $G L_{n}(\mathbf{C})$ is called unipotent if it is contained in a conjugate of the group of upper triangular matrices with all diagonal entries equal to one. Any unipotent subgroup is nilpotent; hence, a finitely-generated unipotent subgroup is polycyclic and torsion-free. It is well known that a polycyclic group has finite cohomological dimension if and only if it is torsion-free; moreover, the cohomological dimension is the same as the Hirsch rank. For a solvable group $\Gamma$ with solvable series, $1=\Gamma_{n}<\Gamma_{n-1}<\cdots<\Gamma_{1}=\Gamma$, the Hirsch rank, $h(\Gamma)=$ $\sum_{i=1}^{n-1} \operatorname{dim}_{\mathrm{Q}}\left(\Gamma_{i} / \Gamma_{i+1} \otimes \mathrm{Q}\right)$, is independent of the choice of solvable series; thus, for a polycyclic group $\Gamma, h(\Gamma)$ is the number of infinite factors in a normal series with cyclic quotients.

We announce our main result.

THEOREM. Let $A$ be a finitely-generated integral domain of characteristic zero. A group $\Gamma \subset G L_{n}(A), n>0$, has finite $V C D$ if and only if there is a finite upper bound on the Hirsch ranks of its finitely-generated unipotent subgroups.

We obtain easily the following curious corollary.

COROLLARY 1. Every finitely-generated subgroup of the unitary group $U_{n}(\mathrm{C}), n>0$, has finite virtual cohomological dimension.

The following result is immediate; it, however, was original motivation for our Theorem.

Received by the editors December $10,1980$.

1980 Mathematics Subject Classification. Primary 20J05; Secondary 20H20.

${ }^{1}$ Research supported by the National Science Foundation.

(ㄷ) 1981 Amerizan Mathematical Society $0002-9904 / 81 / 0000-0207 / \$ 01.75$ 
Corollary 2 (SERRE [3, ThÉorÈme 5]). Every finitely-generated subgroup of $G L_{n}(\mathbf{Q}), n>0$, has finite virtual cohomological dimension.

Ralph Strebel has suggested, as a consequence of our Theorem, that we generalize certain results of Bieri. Before mentioning that generalization, we require the following corollary.

Corollary 3. Let $F$ denote a field of characteristic zero. If $\Gamma$ is a finitely-generated subgroup of $G L_{n}(F), n>0$, with center $Z$ then $\Gamma$ has finite $V C D$ if and only if $Z$ and $\Gamma / Z$ have finite $V C D$.

A group $\Gamma$ is said to be of type FP if the trivial $\Gamma$-module $\mathbf{Z}$ has a finite resolution by finitely-generated projective $\mathrm{Z \Gamma}$ modules. Combining our Corollary 3 with the methods of Bieri [2] we obtain the following as an immediate consequence.

COROllary 4. If $\Gamma$ is of type FP and has a faithful linear representation over a field of characteristic zero then the center of $\Gamma$ is finitely generated.

The proof of our main theorem involves the action of linear groups on the Tits' buildings for discretely-valued fields. This ingredient already occurs in Serre [3]. Serre's application to groups of type FA [4, Proposition 2] was carried further by Bass [1, Theorem 6.5] in describing finitely-generated subgroups of $G L_{2}(C)$. Inspired by this we have shown that (with the notation of the Theorem) there are finitely many valuations $v_{1}, \ldots, v_{m}$ of the quotient field of $A$ such that $A \cap \mathcal{O}_{v_{1}} \cap \cdots \cap \mathcal{O}_{v_{m}}$ is the ring of integers in a number field $K$. This is used to produce an action of $\Gamma$ on a contractible cell complex which is a product of finitely many Tits' buildings, such that the stabilizer of each cell consists of matrices whose characteristic roots are algebraic integers in an extension of $K$ having bounded degree. Under the hypothesis of the Theorem, one can bound the virtual cohomological dimensions of these stabilizers by representing them as discrete subgroups of Lie groups.

A result due to Quillen [3, Proposition 2] then implies that $\Gamma$ has finite VCD. The details of proof will appear elsewhere; the techniques can be further refined to give a theory of hierarchies for matrix groups which is analogous to the Haken-Waldhausen theory for 3-manifolds.

We would like to thank Hyman Bass and Ken Brown for their encouragement throughout the stages of development of these results.

\section{BIBLIOGRAPHY}

1. H. Bass, Groups of integral representation type, Pacific J. Math. 86 (1980), 15-52.

2. R. Bieri, A connection between the integral homology and the centre of a rational linear group, Math. Z. 170 (1980), 263-266. 
3. J.-P. Serre, Cohomologie des groupes discrets, Prospects in Mathematics, Princeton Univ. Press, Princeton, N. J., 1971.

4. - Amalgames et points fixes, Proc. Second Internat. Conf. on the Theory of Groups (Australian Nat. Univ., Canberra, 1973), Lecture Notes in Math., Vol. 372, Springer, Berlin, 1974, pp. 633-640.

DEPARTMENT OF MATHEMATICS, UNIVERSITY OF OKLAHOMA, NORMAN, OKLAHOMA 73019

DEPARTMENT OF MATHEMATICS, RICE UNIVERSITY, HOUSTON, TEXAS 77001 